\title{
Size matters: The width and location of a ureteral stone accurately predict the chance of spontaneous passage
}

\author{
Johan Jendeberg $^{1}$ (D) - Håkan Geijer ${ }^{1}$ - Muhammed Alshamari ${ }^{1} \cdot$ Bartosz Cierzniak $^{2}$. \\ Mats Lidén ${ }^{1}$
}

Received: 8 July 2016 /Revised: 13 March 2017 / Accepted: 12 April 2017 /Published online: 7 June 2017

(C) The Author(s) 2017. This article is an open access publication

\begin{abstract}
Objectives To determine how to most accurately predict the chance of spontaneous passage of a ureteral stone using information in the diagnostic non-enhanced computed tomography (NECT) and to create predictive models with smaller stone size intervals than previously possible.

Methods Retrospectively 392 consecutive patients with ureteric stone on NECT were included. Three radiologists independently measured the stone size. Stone location, side, hydronephrosis, CRP, medical expulsion therapy (MET) and all follow-up radiology until stone expulsion or 26 weeks were recorded. Logistic regressions were performed with spontaneous stone passage in 4 weeks and 20 weeks as the dependent variable.

Results The spontaneous passage rate in 20 weeks was 312 out of 392 stones, $98 \%$ in $0-2 \mathrm{~mm}, 98 \%$ in $3 \mathrm{~mm}, 81 \%$ in $4 \mathrm{~mm}, 65 \%$ in $5 \mathrm{~mm}, 33 \%$ in $6 \mathrm{~mm}$ and $9 \%$ in $\geq 6.5 \mathrm{~mm}$ wide stones.

The stone size and location predicted spontaneous ureteric stone passage. The side and the grade of hydronephrosis only predicted stone passage in specific subgroups.

Conclusion Spontaneous passage of a ureteral stone can be predicted with high accuracy with the information available in the NECT. We present a prediction method based on stone size and location.
\end{abstract}

Johan Jendeberg

johan.jendeberg@regionorebrolan.se

1 Department of Radiology, Faculty of Medicine and Health, Örebro University Hospital, 70185 Örebro, Sweden

2 Department of Surgery, Faculty of Medicine and Health, Örebro University, Örebro, Sweden
Key Points

- Non-enhanced computed tomography can predict the outcome of ureteral stones.

- Stone size and location are the most important predictors of spontaneous passage.

- Prediction models based on stone width or length and stone location are introduced.

- The observed passage rates for stone size in mm-intervals are reported.

- Clinicians can make better decisions about treatment.

Keywords Spiral computed tomography · Ureteral calculi · Kidney stone $\cdot$ Ureter $\cdot$ Renal colic

$\begin{array}{ll}\text { Abbreviations } \\ \text { AUA } & \text { American Urological Association } \\ \text { AUC } & \text { Area under the curve } \\ \text { CRP } & \text { C-reactive protein } \\ \text { EAU } & \text { European Association of Urology } \\ \text { ESWL } & \text { Extracorporeal shock-wave lithotripsy } \\ \text { IVU } & \text { Intravenous urography } \\ \text { KUB } & \text { Kidney-Ureter-Bladder abdominal radiography } \\ \text { MET } & \text { Medical expulsion therapy } \\ \text { MPR } & \text { Multiplanar reformats } \\ \text { NECT } & \text { Non-enhanced computed tomography } \\ \text { PACS } & \text { Picture archiving and communication system } \\ \text { RIS } & \text { Radiology information system } \\ \text { UVJ } & \text { Ureterovesical junction }\end{array}$

\section{Introduction}

Urolithiasis is a common cause of acute flank pain with increasing prevalence and increasing costs for health systems [1, 2]. 
According to earlier studies [3-5] 75-90\% of stones in the ureter pass spontaneously. If the stone can be expected to pass spontaneously within a reasonable time and the pain is tolerable, the first approach is watchful waiting, with or without accompanying medical expulsive therapy (MET) [6]. Stones that are not expected to pass are treated with extracorporeal shock wave lithotripsy (ESWL), laser lithotripsy or percutaneous stone extraction via the renal pelvis. There are risks with both the conservative and the invasive approaches, such as sepsis that can occur either because of an obstructing stone or as a post-procedure complication. The main risk of the conservative approach is failure and that the patient has had to endure symptoms to no benefit. The major risk of intervention is that it was unnecessary, exposing the patient to the potential risks of, for example, anaesthesia, upper urinary tract infections and ureteral injury. Thus prediction of the chance for a stone to pass spontaneously is crucial for the appropriate selection of a treatment strategy [7].

It is widely agreed that there is a strong correlation between stone size and location and the likelihood for spontaneous stone passage [3, 4]. More recent studies have suggested other predictive factors, e.g. C-reactive protein (CRP), hydronephrosis [8-11] and side of the stone [12].

There is, however, still no standardized method of stone size measurement, with the most widely used method for diagnosing ureteral stones being non-contrast-enhanced computed tomography (NECT). The uncertainty of stone size measurements includes whether the length or width of the ureteral stone predicts the probability for spontaneous passage. A recent study revealed that 2D measurements underestimate the stone size compared to 3D measurements [13]. Due to the non-standardization of stone size measurement, the meta-analysis published in the 2007 Guidelines from the American Urological Association (AUA) and the European Association of Urology (EAU) have large intervals $-68 \%$ of stones $<5 \mathrm{~mm}$ and $47 \%$ of stones $>5 \mathrm{~mm}$ pass spontaneously [6]. Consequently the guidelines state that watchful waiting is an optional initial approach for ureteral stones $<10 \mathrm{~mm}$. These recommendations were not changed in the most recent guidelines from EAU [14].

Thus, the aim of the present study was to determine how to most accurately predict the chance of spontaneous passage of a ureteral stone using the information available in the diagnostic NECT, including reformats in the three most commonly used image planes with clearly defined measurements and to create predictive models for spontaneous stone passage.

\section{Materials and methods}

\section{Patient population}

The Regional Research Ethics Board approved this retrospective study and waived informed consent. We retrospectively reviewed 1,824 consecutive low-dose NECTs performed between April 2012 and September 2014 in patients who presented at our emergency department with suspected renal colic. The sample size of $350-400$ patients was estimated with the objective to reach a width of a $95 \%$ confidence interval (CI) for the proportion of passed stones of \pm 10 percentage points. 392 patients were found to have a solitary stone $>2 \mathrm{~mm}$ (measured in the axial plane) in the ureter and were included in the study. Patient characteristics are shown in Table 1. Exclusion criteria and numbers are shown in Fig. 1.

\section{CT protocol}

The CT examinations were performed on two different CT scanners: 166 patients were examined using a 40-detector row CT scanner (Brilliance, Philips Medical Systems, Best, The Netherlands) with a low-dose NECT protocol for the urinary tract $(120 \mathrm{kV}, 70 \mathrm{mAs} / \mathrm{slice}$, CTDI $4.9 \mathrm{mGy}, 40 \times$ $0.625 \mathrm{~mm}$, standard filter [B], supine position). 226 patients were examined with a $2 \times 128$-channel scanner (Somatom Definition Flash, Siemens, Erlangen, Germany) (120 kVp, $70 \mathrm{mAs} / \mathrm{slice}$ CTDI $4.7 \mathrm{mGy} 128 \times 0.6 \mathrm{~mm}$, filter B20f, B25f or I30f, supine position). Three- or 5-mm axial, coronal and sagittal multiplanar reformats (MPR) in the main axes of the patient were generated.

\section{Image review and patient data}

The calculi were independently measured by three radiologists (with 25 (HG), 11 (JJ) and 9 (MA) years of experience each, respectively, in reading abdominal $\mathrm{CT}$ ) with the integrated PACS measurement tool (Sectra IDS7, Linköping, Sweden). No training for consensus measurement between the readers was performed. The readers were not blinded to the initial report. The largest stone diameter was measured in each of the three reformations (axial, coronal and sagittal) relative to the main axes of the patients' body, in a standardized bone window setting (L300/W1120) and in a standardized soft tissue window setting (L50/W400) at a fixed zoom level of (pixel to pixel) $\times 8$ [15]. The measurements were reported in $\mathrm{mm}$ to 1 decimal point. The length of the calculi was defined as the largest of the three measurements and the width as the smallest (Fig. 2). [13] The mean value of the three readers' estimations was used in the study. If a reader could not see the calculus in one reformation, this was reported as $0 \mathrm{~mm}$.

Stone location was defined as upper if the stone was located cranial to the sacroiliac joint, midureter (mid) overlying the sacroiliac joint, distal to the sacroiliac joint (dist) or at the ureterovesical junction (UVJ). Subsequently, the three groups overlying the sacroiliac joint and distal were grouped giving an upper and a lower (mid, dist and UVJ) position.

The presence of hydronephrosis was graded $0-4(0=$ no, $1=$ mild, $2=$ moderate, $3=$ pronounced, $4=$ massive) by read er 1 (JJ) [16]. 
Table 1 Comparison of patients according to spontaneous passage of stone

\begin{tabular}{|c|c|c|c|c|c|}
\hline & \multirow[t]{3}{*}{ All $(\mathrm{n}=392)$} & \multicolumn{2}{|c|}{ Short-term outcome } & \multicolumn{2}{|c|}{ Long-term outcome } \\
\hline & & \multicolumn{2}{|l|}{$(\mathrm{n}=220)$} & \multicolumn{2}{|l|}{$(\mathrm{n}=392)$} \\
\hline & & $(\%)$ & & $(\%)$ & \\
\hline & & Passage & Non-passage & Passage & Non-passage \\
\hline & & $\mathrm{n}=166$ & $\mathrm{n}=54$ & $\mathrm{n}=312$ & $\mathrm{n}=80$ \\
\hline Age, y $( \pm \mathrm{SD})$ & $50( \pm 16)$ & $49( \pm 15)$ & $52( \pm 17)$ & $49( \pm 16)$ & $54( \pm 16)$ \\
\hline Range & $18-100$ & $18-84$ & $19-100$ & $18-84$ & $25-100$ \\
\hline \multicolumn{6}{|l|}{ Sex } \\
\hline Male & $290(74 \%)$ & $120(72 \%)$ & $43(80 \%)$ & $234(75 \%)$ & $56(70 \%)$ \\
\hline Female & $102(26 \%)$ & $46(28 \%)$ & $11(20 \%)$ & $78(25 \%)$ & $24(30 \%)$ \\
\hline \multicolumn{6}{|l|}{ Side (R to $\mathrm{L}$ ) } \\
\hline Right & $179(46 \%)$ & $73(44 \%)$ & $29(54 \%)$ & $137(44 \%)$ & $42(52 \%)$ \\
\hline Left & $213(54 \%)$ & $93(56 \%)$ & $25(46 \%)$ & $175(56 \%)$ & $38(48 \%)$ \\
\hline Width, mm $( \pm \mathrm{SD})$ & $3.7( \pm 1.6)$ & $3.2 \pm 1.1$ & $4.9( \pm 1.5)$ & $3.2( \pm 1.1)$ & $5.7( \pm 1.7)$ \\
\hline Range & $0.5-10.3$ & $0.5-7.7$ & $1.7-10.3$ & $0.5-7.7$ & $1.7-10.3$ \\
\hline \multicolumn{6}{|l|}{ Location } \\
\hline Upper ureter & $124(32 \%)$ & $32(19 \%)$ & $29(54 \%)$ & $68(22 \%)$ & $56(70 \%)$ \\
\hline Mid ureter & $37(9 \%)$ & $13(8 \%)$ & $8(15 \%)$ & $29(9 \%)$ & $8(10 \%)$ \\
\hline Distal ureter & $122(31 \%)$ & $59(36 \%)$ & $11(20 \%)$ & $112(36 \%)$ & $10(13 \%)$ \\
\hline UVJ & $109(28 \%)$ & $62(37 \%)$ & $6(11 \%)$ & $103(33 \%)$ & $6(7 \%)$ \\
\hline \multicolumn{6}{|l|}{ Hydronephrosis } \\
\hline Grade 3 & $51(13 \%)$ & $11(7 \%)$ & $8(15 \%)$ & $29(9 \%)$ & $22(27 \%)$ \\
\hline Grade 2 & $196(50 \%)$ & $95(57 \%)$ & $21(39 \%)$ & $159(51 \%)$ & $37(46 \%)$ \\
\hline Grade 1 & $107(27 \%)$ & $37(22 \%)$ & $22(41 \%)$ & $88(28 \%)$ & $19(24 \%)$ \\
\hline None & $38(10 \%)$ & $23(14 \%)$ & $3(6 \%)$ & $36(12 \%)$ & $2(3 \%)$ \\
\hline CRP mean $( \pm \mathrm{SD})$ & $8.6( \pm 34)$ & $5.7( \pm 10.7)$ & $6.0( \pm 11.1)$ & $7.5( \pm 34.8)$ & $13.1( \pm 30)$ \\
\hline \multirow[t]{2}{*}{ Range } & $1-586$ & $1-81$ & $1-65$ & $1-586$ & $1-175$ \\
\hline & (11 missing) & (5 missing) & (3 missing) & (7 missing) & \\
\hline \multirow[t]{2}{*}{ MET } & $109(28 \%)$ & $35(21 \%)$ & $16(30 \%)$ & $79(25 \%)$ & $30(38 \%)$ \\
\hline & (1 missing) & & & (1 missing) & \\
\hline Spontaneous passage & $312(80 \%)$ & & & & \\
\hline Intervention & $73(19 \%)$ & & & & \\
\hline No passage or intervention & $7(2 \%)$ & & & & \\
\hline
\end{tabular}

Width stone width (bone window (L300/W1120)), CRP C-reactive protein, MET medical expulsive therapy

The side of the stone (right/left), age and sex of the patient were recorded. CRP levels at the time of the primary NECT and whether the patient had been prescribed MET (alfuzosin $10 \mathrm{mg} \times 1$ for 30 days) was recorded from the patient's medical chart.

\section{Outcome measure - spontaneous passage of stone}

All consecutive radiological examinations in RIS/PACS regarding stone passage and intervention were reviewed up to 26 weeks after the diagnostic NECT. Observed stone passage was defined as presence of follow-up radiological examination (CT or intravenous urography (IVU)) where a ureteral stone was definitely not present anymore. Stone passage was defined as spontaneous if conservative treatment, with or without analgesic or MET, led to stone passage. Any intervention, such as nephropyelostomy, ESWL or ureteroscopy, was recorded as non-spontaneous passage of stone observed at the first day of intervention. The 312 stones with spontaneous passage were verified with IVU $(n=239)$, NECT $(n=70)$ or contrast-enhanced CT (CECT) $(n=3)$.

\section{Short-term versus long-term outcome}

Due to the retrospective nature of the study, the follow-up intervals were non-standardized. Generally the follow-up 
Fig. 1 Flowchart showing exclusion criteria and numbers

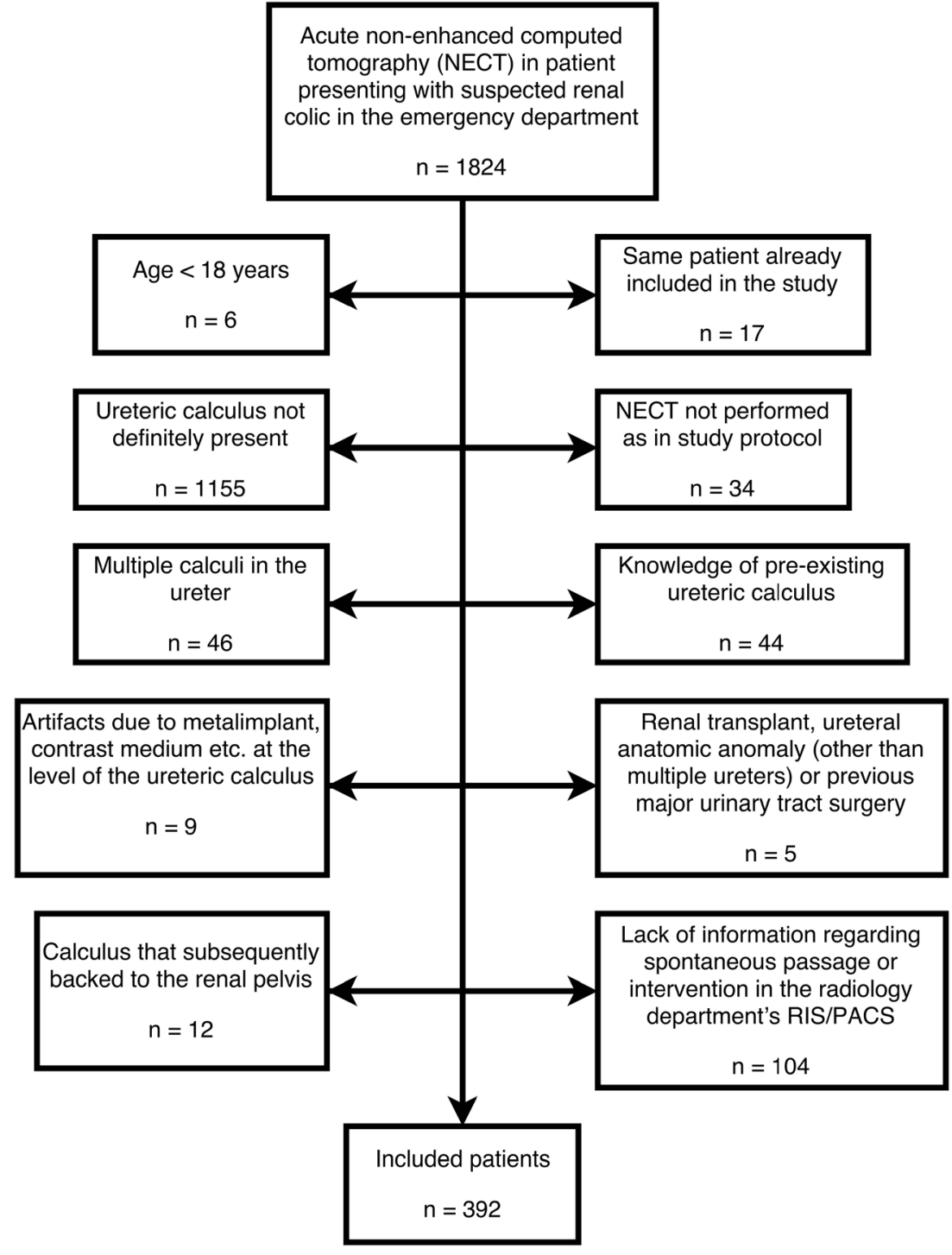

examination was performed in 4-6 weeks after the first event if watchful waiting was used, irrespective of whether the symptoms resolved or the patient passed and retrieved a stone. In order to achieve an unbiased estimation of the passage rates within approximately 4 weeks, we created a short-term subgroup including patients with the first follow-up examination $28 \pm 14$ days after the diagnostic NECT. The outcome measure (passed/not passed) in the short-term cohort was obtained from the follow-up examination performed closest to day 28 . All patients were included in the long-term follow-up, where the outcome measure was obtained from the follow-up examination closest to 140 days (20 weeks).

\section{Statistical analyses}

The statistical analysis was performed using IBM SPSS for Mac OS v24.0.0.0 (SPSS Inc., Chicago, Il, USA).
Multivariate logistic regression was performed with spontaneous stone passage as the dependent variable and with independent variables as shown in Table 2. Addition of quadratic terms in the multiple regression and visual examination of the predictive curves versus observed passage rates showed no evidence of non-linearity.

Before stepwise regression, collinearity between predictors was assessed with the Spearman correlation coefficient. Because of the high correlation between the stone length and stone width, the length was excluded from the stepwise regression. The other predictors showed a low correlation $(|\mathrm{r}|<0.5)$, and were included in the further analysis. Automated stepwise backward logistic regression was performed in the full cohort and in the subgroups upper and lower stones. Predictive univariate logistic regression curves based on stone width and length were created for the subgroups upper and lower stones in the short and long 

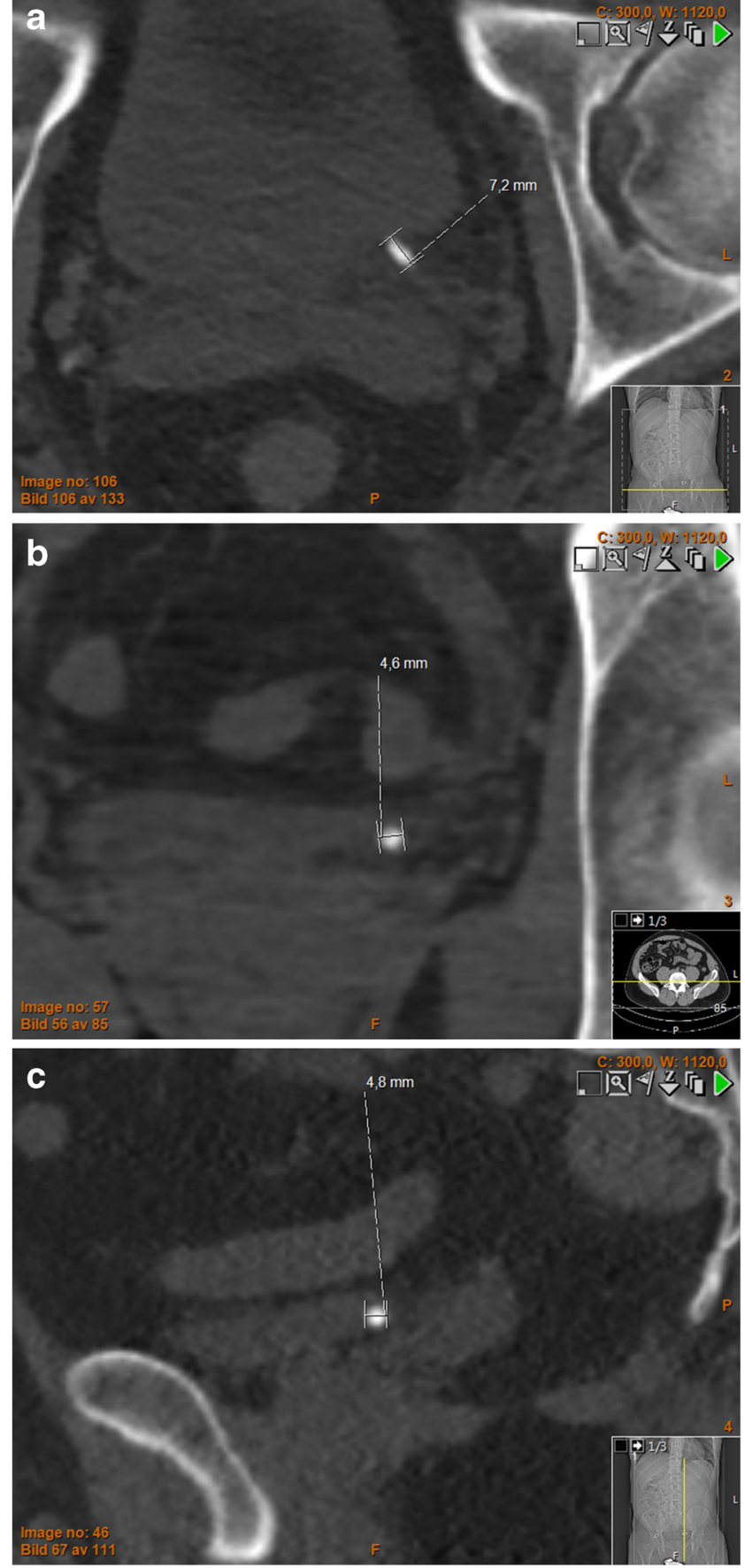

Fig. 2 Distal ureteral stone: (a) axial, (b) coronal, (c) sagittal. Nonenhanced computed tomography of the urinary tract, window settings L300/W1120, showing a distal ureteral stone in a 39-year-old male. In each of the three reformats the largest diameter is measured. Stone length $=$ the largest of these measurements $(\max [\mathrm{ax}, \mathrm{cor}, \mathrm{sag}])$. Stone width $=$ the smallest of these measurements $(\min [\mathrm{ax}, \mathrm{cor}, \mathrm{sag}])$. Here the stone length $=7.2 \mathrm{~mm}$ and stone width $=4.6 \mathrm{~mm}$

term (Figs. 3, 4, 5 and 6), for measurements in the bone and soft tissue window separately.

Receiver operating characteristic (ROC) analysis was performed for the predictive regression models.

\section{Results}

Spontaneous stone passage was observed in 312 patients $(80 \%)$. Intervention was performed in 73 patients $(19 \%)$ in the 20-week follow-up, mean intervention day 37 (range 0 179). Seven patients without spontaneous passage in 20 weeks did not undergo any intervention during the study period. The mean \pm 1 SD outcome observation day in the short-term group was $31 \pm 7$. The observed spontaneous stone passage rate at different stone width, measured in bone window (L300/W1120), and in different locations is listed in Table 3 with 95\% CIs for proportions using an exact method. Using the bone window the Bland Altman 95\% limits of agreement for the width estimation between reader 1 and 2 was $0.7 \pm$ $1.3 \mathrm{~mm}$, between reader 1 and $3,0.7 \pm 1.3 \mathrm{~mm}$ and between reader 2 and 3, $0.1 \pm 1.1 \mathrm{~mm}$. Using the soft tissue window the Bland Altman 95\% limits of agreement for the width estimation between reader 1 and 2 was $0.8 \pm 1.1 \mathrm{~mm}$, between reader 1 and 3, $0.5 \pm 1.1 \mathrm{~mm}$ and between reader 2 and 3, $0.4 \pm$ $0.9 \mathrm{~mm}[17]$.

\section{Multivariate logistic regression}

The multivariate logistic regression analyses were performed using the bone window measurements. The width and length of the ureteral stones were highly correlated (correlation coefficient 0.96). In a univariate logistic regression using width and length as predictor variable the AUCs were similar, 0.90 and 0.89 , respectively, for long-term outcome.

In the multivariate logistic regression analysis the stone width took precedence over the stone length, both in shortterm (width $p=0.038$, length $p=0.39$ ) and long-term follow up (width $p<0.001$, length $p=0.54$ ) (see Table 2). The problem of collinearity between the predictor variables, which made them unsuitable for simultaneous use in a multivariate model, was solved by removing the stone length in the further analysis.

The stone location was a significant predictor of stone passage. As there were relatively few stones localized in the midureter (37/392, Table 1), and the odds ratio was similar in the mid, dist and UVJ locations (Table 2), the latter three were grouped as lower stones as described earlier. Due to the lack of significant predictive differences between the initially chosen grades of hydronephrosis, this was regrouped into low-grade (grade 0-1) and high-grade (grade 2-4) hydronephrosis in the further analysis.

\section{Stepwise backwards logistic regression}

According to position the stones were divided in the two subgroups, upper and lower stones.

Multivariate stepwise backwards logistic regression was performed in the full cohort and in these subgroups. In upper 
Table 2 Multivariate logistic regression with all independent variables. Odds ratios (ORs) for stone passage with $95 \%$ confidence intervals (CIs)

\begin{tabular}{|c|c|c|c|c|c|c|}
\hline & \multicolumn{3}{|c|}{ Short-term outcome } & \multicolumn{3}{|c|}{ Long-term outcome } \\
\hline & OR & $95 \% \mathrm{CI}$ & $p$ & OR & $95 \% \mathrm{CI}$ & $p$ \\
\hline Width & 0.40 & $0.17-0.95$ & 0.038 & 0.31 & $0.16-0.58$ & $<0.001$ \\
\hline Length & 0.76 & $0.40-1.4$ & 0.39 & 0.86 & $0.54-1.4$ & 0.54 \\
\hline \multicolumn{7}{|l|}{ Position vs. Upper* } \\
\hline Midureter & 2.0 & $0.49-8.5$ & 0.33 & 4.3 & $1.2-15$ & 0.023 \\
\hline Distal & 4.5 & $1.4-14$ & 0.01 & 7.7 & $2.7-22$ & $<0.001$ \\
\hline UVJ & 5.4 & $1.6-18$ & 0.006 & 3.3 & $1.1-10$ & 0.032 \\
\hline \multicolumn{7}{|c|}{ Hydronephrosis vs. no hydronephrosis* } \\
\hline Grade 1 & 0.21 & $0.035-1.3$ & 0.087 & 0.24 & $0.03-2.2$ & 0.21 \\
\hline Grade 2 & 1.6 & $0.27-9.7$ & 0.60 & 0.60 & $0.07-5.3$ & 0.65 \\
\hline Grade 3 & 0.86 & $0.10-6.9$ & 0.89 & 0.38 & $0.04-3.6$ & 0.40 \\
\hline Age & 1.0 & $0.97-1.0$ & 0.92 & 0.98 & $0.96-1.0$ & 0.18 \\
\hline Sex (f vs. m) & 1.5 & $0.52-4.6$ & 0.43 & 0.91 & $0.39-2.1$ & 0.83 \\
\hline Side (left vs. right) & 1.9 & $0.76-4.7$ & 0.17 & 2.9 & $1.3-6.4$ & 0.007 \\
\hline CRP & 1.0 & $0.98-1.1$ & 0.21 & 0.99 & $0.99-1.0$ & 0.27 \\
\hline MET & 0.94 & $0.33-2.7$ & 0.91 & 0.70 & $0.32-1.5$ & 0.36 \\
\hline Constant & 149 & & 0.001 & 2609 & & $<0.001$ \\
\hline
\end{tabular}

*Categorical variable

An OR close to 1 indicates that the variable does not affect the probability of spontaneous stone passage. An OR $>1$ indicates that this variable is associated with higher probability and an $\mathrm{OR}<1$ that this variable is associated with lower probability of spontaneous stone passage stones stone width and side (right vs. left) remained significant predictors of stone passage in the long-term, but in the shortterm only stone width was a significant predictor (Table 4).

In lower stones the side and hydronephrosis (low vs. high grade) remained significant predictors of stone passage in the short term in addition to stone width. Leftsided stones had a higher probability of passing spontaneously than right-sided stones. Stones causing moderate to massive hydronephrosis had a higher probability of passing spontaneously than stones causing no or only mild hydronephrosis. In the subgroup long-term follow-up lower stones, only stone width was an unambiguous significant predictor (Table 4). Although not excluded by the stepwise regression, the side and age had $p>0.05$. Age had an odds ratio of 0.97 , indicating minimal influence on the probability of spontaneous passage.
Fig. 3 Probability for spontaneous stone passage as a function of stone width, bone window (L300/W1120). (a) Upper ureteral stones (univariate logistic regression curves). Error bars showing observed long-term 95\% confidence intervals. AUC for the prediction short term: 0.92 , long term: 0.93. (b) Lower ureteral stones (univariate logistic regression curves). Error bars showing observed long-term $95 \%$ confidence intervals. AUC for the short term prediction: 0.80 , long term prediction: 0.83
Prediction of stone passage - bone window, stone width

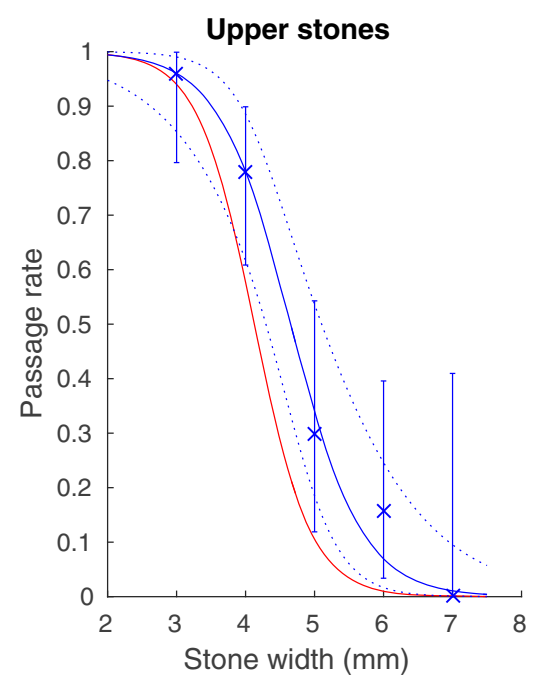


Fig. 4 Probability for spontaneous stone passage as a function of stone width, soft tissue window (L50/W400). (a) Upper ureteral stones (univariate logistic regression curves). Error bars showing observed long-term 95\% confidence intervals. AUC for the short term prediction: 0.92 long term prediction: 0.93. (b) Lower ureteral stones (univariate logistic regression curves). Error bars showing observed long-term 95\% confidence intervals. AUC for the short term prediction: 0.81 , long term prediction: 0.82
Prediction of stone passage - soft tissue window, stone width

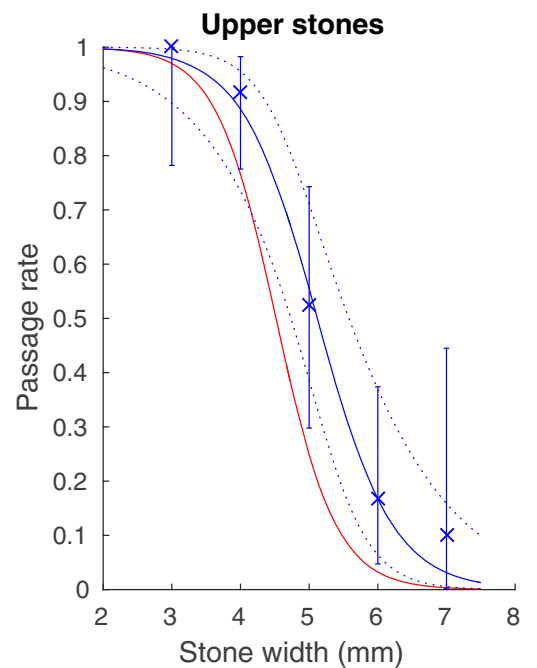

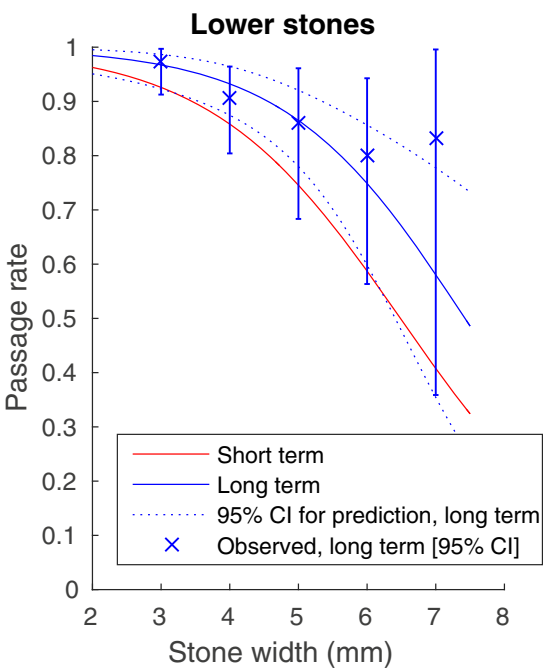

Sex, CRP and MET were not independent predictors of stone passage.

\section{Predictive logistic regression models}

After the stepwise backwards exclusion of the non-significant independent predictor variables predictive univariate logistic regression models were created for the subgroups upper and lower ureteral stones with the predictor variables stone width and length separately for the bone window and for the soft tissue window. Neither stone side nor hydronephrosis were included in the predictive logistic regression models, due to the low number of stones in each subgroup and consequently broad CIs they would cause and because they were not significant predictors in all parts of the ureter. Furthermore, earlier studies on hydronephrosis and side regarding stone passage have shown divergent results $[8,9,12,18]$.
As seen in Figs. 3, 4, 5 and 6, there is a steep middle part of the logistic regression model curves, especially for the upper stones, making the estimated probability for passage range from about $80 \%$ in a $4-\mathrm{mm}$ wide stone, to about $10 \%$ in a 6 $\mathrm{mm}$ wide stone in the long-term follow-up bone window.

\section{Discussion}

Ever since NECT replaced KUB in the 1990s as the primary tool for diagnosing ureteral stones there have been controversies concerning how to measure the ureteral stones for prediction of stone passage rate. A pivotal aspect has been the diversity and sometimes lack of definitions of stone width and length $[5,10-12,15,19-26]$.

We created logistic regression models for prediction of spontaneous passage using a clear definition of the stone
Fig. 5 Probability for spontaneous stone passage as a function of stone length, bone window (L300/W1120). (a) Upper ureteral stones (univariate logistic regression curves). Error bars showing observed long-term $95 \%$ confidence intervals. AUC for the short term prediction: 0.89 , long term prediction: 0.89 . (b) Lower ureteral stones (univariate logistic regression curves). Error bars showing observed long-term $95 \%$ confidence intervals. AUC for the short term prediction: 0.79 , long term prediction: 0.83
Prediction of stone passage - bone window, stone length
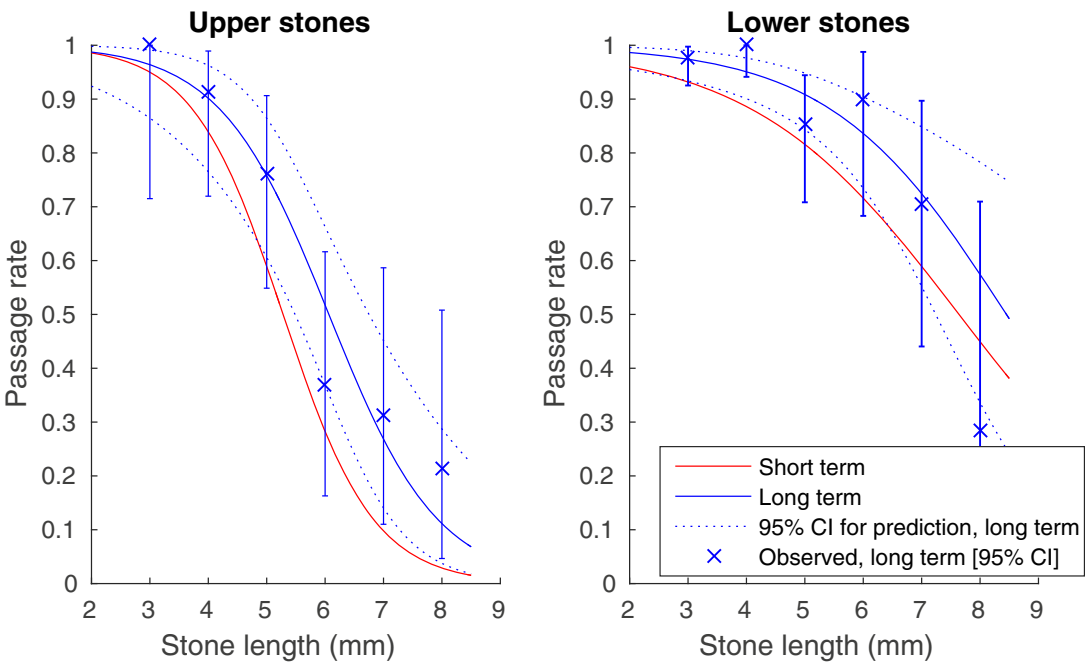
Fig. 6 Probability for spontaneous stone passage as a function of stone length, soft tissue window (L50/W400). (a) Upper ureteral stones (univariate logistic regression curves). Error bars showing observed long-term $95 \%$ confidence intervals. AUC for the short term prediction: 0.90 , long term prediction: 0.91. (b) Lower ureteral stones (univariate logistic regression curves). Error bars showing observed long-term 95\% confidence intervals. AUC for the short term prediction: 0.80 , long term prediction: 0.82
Prediction of stone passage - soft tissue window, stone length

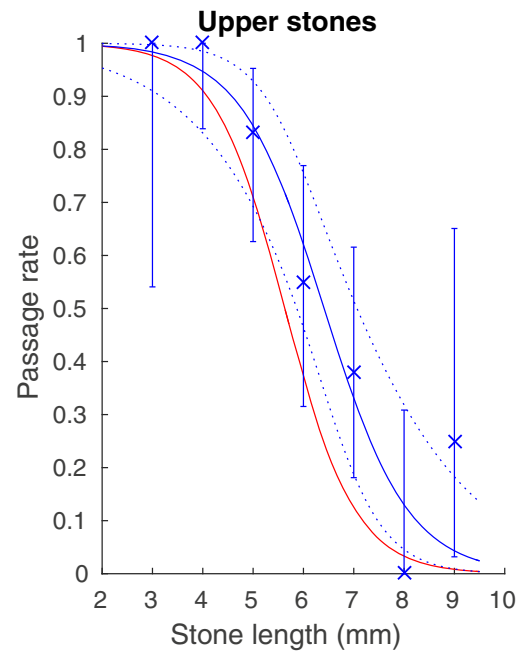

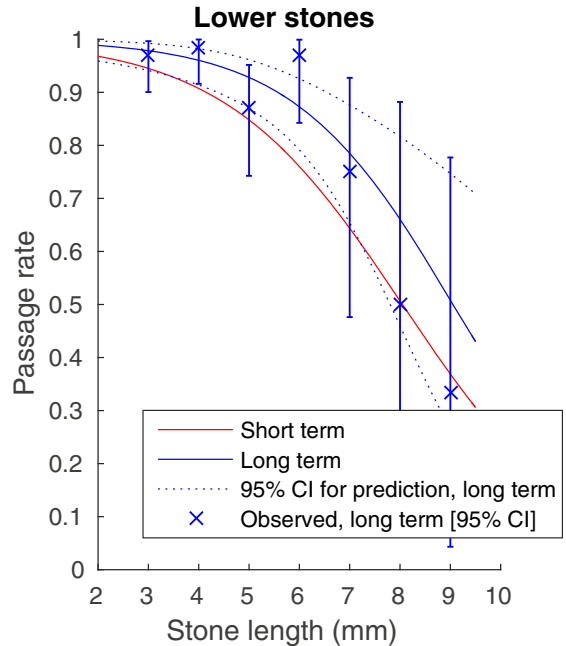

width and length. The strong correlation between the width and the length of a ureteral stone suggests that either measurement can be used in a predictive model with a similar AUC. Thus the choice between the width and the length as a

Table 3 Stone passage rate with 95\% confidence (CIs) and exact numbers depending on stone width, measured in a standardized bone window (L300/W1120)

Stone passage rate

$[95 \% \mathrm{CI}]$

(passed stones/all stones)

\begin{tabular}{|c|c|c|c|c|c|c|c|c|}
\hline \multirow[t]{3}{*}{ Stone width (mm) } & \multicolumn{2}{|l|}{ All stones } & \multicolumn{2}{|l|}{ Upper stones } & \multicolumn{4}{|l|}{ Lower stones } \\
\hline & Both sides & & Both sides & & Both sides & & Left & Right \\
\hline & Short term & Long term & Short term & Long term & Short term & Long term & Short term & Short term \\
\hline \multirow[t]{3}{*}{$0-2.4$} & 0.98 & 0.98 & 1.00 & 1.00 & 0.98 & 0.97 & 1.00 & 0.95 \\
\hline & {$[0.88-1.00]$} & {$[0.92-1.00]$} & {$[0,40-1,00]$} & {$[0.59-1.00]$} & {$[0.87-1.00]$} & {$[0.91-1.00]$} & {$[0.82-1.00]$} & [0.76-1.00] \\
\hline & $(43 / 44)$ & $(82 / 84)$ & $(4 / 4)$ & $(7 / 7)$ & $(39 / 40)$ & $(75 / 77)$ & $(19 / 19)$ & $(20 / 21)$ \\
\hline \multirow[t]{3}{*}{$2.5-3.4$} & 0.92 & 0.98 & 0.87 & 0.96 & 0.93 & 0.99 & 0.89 & 0.97 \\
\hline & {$[0.83-0.97]$} & [0.94-1.00] & {$[0.60-0.98]$} & {$[0.80-1.00]$} & {$[0.83-0.98]$} & {$[0.94-1.00]$} & {$[0.72-0.98]$} & [0.83-1.00] \\
\hline & $(67 / 73)$ & $(119 / 121)$ & $(13 / 15)$ & $(24 / 25)$ & $(54 / 58)$ & $(95 / 96)$ & $(25 / 28)$ & $(29 / 30)$ \\
\hline \multirow[t]{3}{*}{$3.5-4.4$} & 0.71 & 0.81 & 0.67 & 0.78 & 0.74 & 0.83 & 0.94 & 0.50 \\
\hline & {$[0.57-0.83]$} & {$[0.71-0.89]$} & {$[0.43-0.85]$} & {$[0.61-0.90]$} & {$[0.55-0.88]$} & {$[0.69-0.92]$} & {$[0.71-1.00]$} & [0.23-0.77] \\
\hline & $(37 / 52)$ & $(67 / 83)$ & $(14 / 21)$ & $(28 / 36)$ & $(23 / 31)$ & $(39 / 47)$ & $(16 / 17)$ & $(7 / 14)$ \\
\hline \multirow[t]{3}{*}{$4.5-5.4$} & 0.47 & 0.65 & 0.09 & 0.30 & 0.68 & 0.89 & 0.85 & 0.33 \\
\hline & {$[0.28-0.66]$} & [0.49-0.78] & {$[0.00-0.41]$} & {$[0.12-0.54]$} & {$[0.43-0.87]$} & {$[0.72-0.98]$} & [0.55-0.98] & {$\left[\begin{array}{ll}0.04 & 0.78\end{array}\right]$} \\
\hline & $(14 / 30)$ & $(31 / 48)$ & $(1 / 11)$ & $(6 / 20)$ & $(13 / 19)$ & $(25 / 28)$ & $(11 / 13)$ & $(2 / 6)$ \\
\hline \multirow[t]{3}{*}{$5.5-6.4$} & 0.21 & 0.33 & 0.00 & 0.16 & 0.38 & 0.57 & 0.40 & 0.33 \\
\hline & {$[0.05-0.51]$} & [0.18-0.52] & {$[0.00-0.46]$} & {$[0.03-0.40]$} & [0.09-0.76] & [0.29-0.82] & {$[0.05-0.85]$} & {$[0.01-0.91]$} \\
\hline & $(3 / 14)$ & $(11 / 33)$ & $(0 / 6)$ & $(3 / 19)$ & $(3 / 8)$ & 8/14) & $(2 / 5)$ & $(1 / 3)$ \\
\hline \multirow[t]{3}{*}{$\geq 6.5$} & 0.29 & 0.09 & 0.00 & 0.00 & 0.67 & 0.33 & 1.00 & 0.00 \\
\hline & {$[0.04-0.71]$} & {$[0.01-0.28]$} & {$[0.00-0.60]$} & {$[0.00-0.20]$} & [0.09-0.99] & {$[0.04-0.78]$} & {$[0.16-1.00]$} & {$[0.00-0.98]$} \\
\hline & $(2 / 7)$ & $(2 / 23)$ & $(0 / 4)$ & $(0 / 17)$ & $(2 / 3)$ & $(2 / 6)$ & $(2 / 2)$ & $(0 / 1)$ \\
\hline \multirow[t]{3}{*}{ All sizes } & 0.76 & 0.80 & 0.52 & 0.55 & 0.84 & 0.91 & 0.89 & 0.79 \\
\hline & {$[0.69-0.81]$} & {$[0.75-0.84]$} & {$[0.39-0.65]$} & {$[0.46-0.64]$} & {$[0.78-0.90]$} & [0.87-0.94] & [0.81-0.95] & [0.68-0.87] \\
\hline & $(166 / 220)$ & $(312 / 392)$ & $(32 / 61)$ & $(68 / 124)$ & (134/159) & $(244 / 268)$ & $(75 / 84)$ & $(59 / 75)$ \\
\hline
\end{tabular}


Table 4 Results of stepwise logistic regression with stone width, location (upper vs. lower ureter), side and hydronephrosis (low vs. high grade), age, sex, Creactive protein (CRP) and medical expulsion therapy (MET) as independent variables. The side remained a significant predictor in the long-term upper ureteral stones and, together with hydronephrosis, in the short-term lower stones. Odds ratio (OR) for stone passage with $95 \%$ confidence intervals (CIs)

\begin{tabular}{|c|c|c|c|c|c|c|}
\hline & \multicolumn{3}{|c|}{ Short-term outcome } & \multicolumn{3}{|c|}{ Long-term outcome } \\
\hline & OR & $95 \% \mathrm{CI}$ & $p$ & OR & $95 \% \mathrm{CI}$ & $p$ \\
\hline \multicolumn{7}{|l|}{ All stones } \\
\hline Width & 0.28 & $0.19-0.43$ & $<0.001$ & 0.27 & $0.20-0.38$ & $<0.001$ \\
\hline Location lower vs. upper & 4.5 & $1.9-11$ & 0.001 & 5.2 & $2.5-10.7$ & $<0.001$ \\
\hline Side left vs. right & 2.3 & $0.99-5.5$ & 0.052 & 3.2 & $1.5-6.7$ & 0.003 \\
\hline Hydronephrosis high vs. low grade & 4.7 & $1.9-12$ & 0.001 & N/A & N/A & N/A \\
\hline \multicolumn{7}{|l|}{ Upper stones } \\
\hline Width & 0.092 & $0.027-0.32$ & $<0.001$ & 0.13 & $0.059-0.27$ & $<0.001$ \\
\hline Side left vs. right & $\mathrm{N} / \mathrm{A}$ & N/A & $\mathrm{N} / \mathrm{A}$ & 3.7 & $1.1-13$ & 0.034 \\
\hline \multicolumn{7}{|l|}{ Lower stones } \\
\hline Width & 0.33 & $0.20-0.53$ & $<0.001$ & 0.40 & $0.28-0.57$ & $<0.001$ \\
\hline Side left vs. right & 3.6 & $1.1-11$ & 0.028 & 2.7 & $0.92-7.9$ & 0.071 \\
\hline Hydronephrosis high vs. low grade & 7.0 & $2.1-24$ & 0.002 & N/A & N/A & N/A \\
\hline Age & $\mathrm{N} / \mathrm{A}$ & N/A & N/A & 0.97 & $0.94-1.0$ & 0.052 \\
\hline
\end{tabular}

predictor variable is less important, while it is of utmost importance that the selected measure is used consistently. Although the stone width took precedence over the length in the multivariate regression and measuring the width conforms to the intention in earlier studies, we provide predictive models for both stone width and length (Figs. 3, 4, 5, and 6) $[3,5,18]$. We also recommend a high level of magnification to be used, together with a predefined window setting.

To the best of our knowledge the present study with spontaneous passage as the outcome has included the largest number of ureteral stones since the start of the CT era, which made it possible to create logistic univariate regression models for prediction of passage probabilities given the stone size for different stone locations and with two different window settings of L300/W1120 and L50/W400.

It is widely agreed that the stone location is an important predictor of stone passage [3-5, 12]. Our results suggest a classification of stone location into upper and lower ureteral stones when predicting stone passage.

We demonstrated that left-sided ureteral stones seem to pass significantly more often than right-sided in some analyses (see Table 4). Sfoungaristos et al. suggested that the reason might be that the right ureter is typically adherent to the peritoneum, in contrast to the left ureter, providing a better peristalsis in the left ureter [12].

In the present study lower stones causing moderate to massive hydronephrosis passed significantly more often within 4 weeks than stones causing no or only mild hydronephrosis. In the long term there was no significant difference between the grades of hydronephrosis.

The results of earlier studies on hydronephrosis and side regarding stone passage are divergent [8, 9, 12, 18]. Furthermore the CIs became very broad when hydronephrosis and side were added to the predictive regression models, and we chose not to include either of these variables in the models.

In contrast to the results of previous studies [8, 10, 11], CRP was not an independent predictor of spontaneous ureteral stone passage in our study. We did not find MET to be a significant predictor of stone passage, but our study was not designed to assess the impact of MET on stone passage. Only $29 \%$ of the patients were prescribed MET and the study should primarily be considered as conducted without MET. Until most recently, there seemed to be convincing evidence [27, 28] that MET would facilitate stone passage, but this was contradicted by a large recently published randomized controlled trial [25] that did not find any difference between MET and placebo.

Our study has limitations. As the study was retrospective the clinical management of the patients affected the observations of spontaneous passage. This effect was reduced in the short-term analysis by selecting a subgroup where the first observation of stone status was within approximately 4 weeks. Furthermore, the bone window setting is sensitive to reader variations due to a large part of stone with indeterminate greyscale intensities in the periphery, and the reader variations were large even in the soft tissue window. The variability in the study was reduced by using the mean value taken from three readers. However, the reader variations of any radiologist applying the results will affect the estimated prognosis for the individual patient.

As the study was made retrospectively, the follow-up examinations were not standardized. Conforming to the clinical routines of our urology department, most of the follow-ups were IVUs. Very small or low-density stones that caused no obstruction could possibly be missed using IVU. However, all subsequent radiological examinations were checked for 
possible missed stones with a follow-up time of each patient of at least 6 months and the possibility for misclassification of stone passage based on IVU was therefore considered to be low.

The steep middle part of the predictive regression curve simplifies the decision of treatment strategy based on stone size, but is also sensitive for inter- and intra-observer measuring differences and variances related to scan parameters. Patel et al. showed that interobserver variability could be substantially reduced with an automated volume measurement. Several different promising automated reader-independent measurement methods [15, 29-31] have been proposed and further studies with these methods would be of interest.

In conclusion, our results show that spontaneous passage of a ureteral stone can be predicted with high accuracy with the knowledge of stone width or length and whether the stone is localized cranial to the sacroiliac joint or not, if standardized window settings and magnifications are used. The present study demonstrates a method for predicting the probability for stone passage in the short- and long-term, based on stone size and location.

Acknowledgements Preliminary data have been published as a poster (Prognosis of spontaneous ureteral stone passage - as revealed by CT) at the European Congress of Radiology 2016.

\section{Compliance with ethical standards}

Guarantor The scientific guarantor of this publication is Mats Lidén, Department of Radiology, Faculty of Medicine and Health, Örebro University.

Conflict of interest The authors of this manuscript declare no relationships with any companies whose products or services may be related to the subject matter of the article.

Funding This study has received funding from the Research Committee of Region Örebro County.

Statistics and biometry Anders Magnuson, Clinical Epidemiology and Biostatistics Unit, Faculty of Medicine and Health, Örebro Unversity, kindly provided statistical advice for this manuscript.

Informed consent Written informed consent was waived by the Institutional Review Board.

Ethical approval Institutional Review Board approval was obtained.

$$
\begin{aligned}
& \text { Methodology } \\
& \text { - } \quad \text { retrospective } \\
& \text { - diagnostic or prognostic study / observational } \\
& \text { - } \quad \text { performed at one institution }
\end{aligned}
$$

Open Access This article is distributed under the terms of the Creative Commons Attribution 4.0 International License (http:// creativecommons.org/licenses/by/4.0/), which permits unrestricted use, distribution, and reproduction in any medium, provided you give appropriate credit to the original author(s) and the source, provide a link to the Creative Commons license, and indicate if changes were made.

\section{References}

1. Scales CD, Saigal CS, Hanley JM et al (2014) The impact of unplanned postprocedure visits in the management of patients with urinary stones. Surgery 155:769-775

2. Saigal CS, Joyce G, Timilsina AR (2005) Direct and indirect costs of nephrolithiasis in an employed population: opportunity for disease management? Kidney Int 68:1808-1814

3. Sandegård E (1956) Prognosis of stone in the ureter. Acta Chir Scand Suppl 219:1-67

4. Ueno A, Kawamura T, Ogawa A, Takayasu H (1977) Relation of spontaneous passage of ureteral calculi to size. Urology 10:544546

5. Coll DM, Varanelli MJ, Smith RC (2002) Relationship of spontaneous passage of ureteral calculi to stone size and location as revealed by unenhanced helical CT. AJR Am J Roentgenol 178:101103

6. Preminger GM, Tiselius HG, Assimos DG et al (2007) 2007 Guideline for the management of ureteral calculi. Eur Urol 52: 1610-1631

7. Kambadakone AR, Eisner BH, Catalano OA, Sahani DV (2010) New and evolving concepts in the imaging and management of urolithiasis: urologists' perspective. Radiographics 30:603-623

8. Ozcan C, Aydogdu O, Senocak C et al (2015) Predictive Factors for Spontaneous Stone Passage and the Potential Role of Serum CReactive Protein in Patients with 4 to $10 \mathrm{~mm}$ Distal Ureteral Stones: A Prospective Clinical Study. J Urol. doi:10.1016/j.juro. 2015.04.104

9. Ahmed A, Gabr AH, Emara A-A et al (2015) Factors predicting the spontaneous passage of a ureteric calculus of $\leqslant 10 \mathrm{~mm}$. Arab J Urol 13:84-90

10. Aldaqadossi HA (2013) Stone expulsion rate of small distal ureteric calculi could be predicted with plasma $\mathrm{C}$-reactive protein. Urolithiasis 41:235-239

11. Park CH, Ha JY, Park CH et al (2013) Relationship Between Spontaneous Passage Rates of Ureteral Stones Less Than $8 \mathrm{~mm}$ and Serum C-Reactive Protein Levels and Neutrophil Percentages. Korean J Urol 54:615-618

12. Sfoungaristos S, Kavouras A, Perimenis P (2012) Predictors for spontaneous stone passage in patients with renal colic secondary to ureteral calculi. Int Urol Nephrol 44:71-79

13. Liden M, Thunberg P, Broxvall M, Geijer H (2015) Two- and threedimensional CT measurements of urinary calculi length and width: a comparative study. Acta Radiol 56:487-492

14. Türk C, Petř́ík A, Sarica K et al (2015) EAU Guidelines on Diagnosis and Conservative Management of Urolithiasis. Eur Urol. doi:10.1016/j.eururo.2015.07.040

15. Eisner BH, Kambadakone A, Monga M et al (2009) Computerized tomography magnified bone windows are superior to standard soft tissue windows for accurate measurement of stone size: an in vitro and clinical study. J Urol 181:1710-1715

16. Beetz R, Bökenkamp A, Brandis M et al (2001) Diagnosis of congenital dilatation of the urinary tract. Consensus Group of the Pediatric Nephrology Working Society in cooperation with the Pediatric Urology Working Group of the German Society of Urology and with the Pediatric Urology Working Society i. Urologe A 40:495-507, quiz 508-9 
17. Bland JM, Altman DG (1986) Statistical methods for assessing agreement between two methods of clinical measurement. Lancet 1:307-310

18. Miller OF, Kane CJ (1999) Tlime to stone passage for observed ureteral calculi: A guide for patient education. J Urol 162:688-691

19. Lee SR, Jeon HG, Park DS, Choi YD (2012) Longitudinal stone diameter on coronal reconstruction of computed tomography as a predictor of ureteral stone expulsion in medical expulsive therapy. Urology 80:784-789

20. Berkovitz N, Simanovsky N, Katz R et al (2010) Coronal reconstruction of unenhanced abdominal CT for correct ureteral stone size classification. Eur Radiol 20:1047-1051

21. Nadler RB, Stern JA, Kimm S et al (2004) Coronal imaging to assess urinary tract stone size. J Urol 172:962-964

22. Liden M, Andersson T, Geijer H (2011) Making renal stones change size-impact of $\mathrm{CT}$ image post processing and reader variability. Eur Radiol 21:2218-2225

23. Zorba OU, Ogullar S, Yazar S, Akca G (2015) CT-based determination of ureteral stone volume: A predictor of spontaneous passage. J Endourol. doi:10.1089/end.2015.0481

24. Tiselius H (2003) Stone Burden in an Average Swedish Population of Stone Formers Requiring Active Stone Removal: How Can the Stone Size Be Estimated in the Clinical Routine? Eur Urol 43:275281
25. Pickard R, Starr K, MacLennan G et al (2015) Medical expulsive therapy in adults with ureteric colic: a multicentre, randomised, placebo-controlled trial. Lancet (London, England) 386:341-349

26. Choi T, Yoo KH, Choi S-K et al (2015) Analysis of factors affecting spontaneous expulsion of ureteral stones that may predict unfavorable outcomes during watchful waiting periods: What is the influence of diabetes mellitus on the ureter? Korean J Urol 56:455-460

27. Campschroer T, Zhu Y, Duijvesz D et al (2014) Alpha-blockers as medical expulsive therapy for ureteral stones. Cochrane Database Syst Rev 4, CD008509. doi:10.1002/14651858.CD008509.pub2

28. Seitz C, Liatsikos E, Porpiglia F et al (2009) Medical therapy to facilitate the passage of stones: what is the evidence? Eur Urol 56: 455-471

29. Demehri S, Steigner ML, Sodickson AD et al (2012) CT-based determination of maximum ureteral stone area: a predictor of spontaneous passage. AJR Am J Roentgenol 198:603-608

30. Patel SR, Stanton P, Zelinski N et al (2011) Automated renal stone volume measurement by noncontrast computerized tomography is more reproducible than manual linear size measurement. J Urol 186:2275-2279

31. Liden M, Andersson T, Broxvall M et al (2012) Urinary stone size estimation: a new segmentation algorithm-based CT method. Eur Radiol 22:731-737 